Diabetologia 10, $139-143$ (1974)

(C) by Springer-Verlag 1974

\title{
On Measuring the Adequacy of Diabetes Regulation: Comparison of Continuously Monitored Blood Glucose Patterns with Values at Selected Time Points*
}

\author{
G.D. Molnar, W.F. Taylor and A. Langworthy \\ Mayo Clinic and Mayo Foundation, Rochester, Minnesota \\ Received: August 11, 1972, and in revised form: December 12, 1973
}

Summary. Individual blood glucose (BG) measurements at selected time points were compared with continuously recorded BG data as criteria of the adequacy of diabetes regulation. Indices reflecting the adequacy of diabetes regulation have previously been developed from continuously monitored BG measurements during studies under standardized near-normal living conditions. These indices are: (1) mean amplitude of glycemic excursions (MAGE), (2) diurnal mean blood glucose (MBG), (3) mean of daily differences of paired BG values (MODD). Because of the intensive studies necessary to obtain these indices, approximations based on individual BG measurements which might easily be obtained in practice were sought. The $\mathrm{BG}$ value $80 \mathrm{~min}$ after breakfast correlated best with the MAGE. The average of the fasting BG value and the value at $80 \mathrm{~min}$ after breakfast correlated well with the MBG. These individual BG measurements distinguished the groups of subjects. The difference between fasting $B G$ values on successive days ( $\triangle F B G$ ) correlated well with the MODD. However, unlike MODD itself, $\triangle F B G$ did not distinguish the groups of subjects. Some other selected $\mathrm{BG}$ values with different timing were nearly equally highly correlated with these three criteria of BG behavior. Thus, relatively few but critically timed BG measurements on successive days, with suitable urinary glucose measurements, during standardized therapeutic programs may serve as an index of the efficacy of the therapy. By these same means, the characteristics of the patient's diabetes might also be assessed.

Key words: Blood glucose, continuous blood glucose analysis, diabetes therapy criteria, unstable diabetes.
This report initiates a search for practical applications of the knowledge acquired by continuous blood glucose $(B G)$ monitoring $[1-3]$. In this article we compare individual measurements of $\mathrm{BG}$ with continuously recorded BG data as criteria of the adequacy of diabetes regulation. The individual measurements were selected at time points found to be most useful for gauging the $B G$ variations. In a previous report [4] we analyzed the relationships between amounts of urinary glucose and corresponding BG values determined by a continuous monitoring procedure. Blood and urinary glucose determinations are the most widely used laboratory measures of the adequacy of diabetes regulation [5-10] despite the recognition that disturbances of metabolism of carbohydrates other than glucose, and of protein and fat as well, are part of the biochemical derangements in diabetes mellitus. Glucose measurements are more generally available as well as more readily interpretable than other measurements.

To identify and quantify several aspects of a patient's BG regulatory stability, we have previously analyzed the results of our continuous BG monitorings [1] in terms of (1) the extent of within-day BG fluctuations (the mean amplitude of BG excursions: MAGE) [2], (2) between-day BG variability (the mean of daily differences in matched $\mathrm{BG}$ values: MODD) [3], and (3) overall glycemia (the mean $B G$ level: MBG). These

* This investigation was supported in part by Research Grant AM-10152 from the National Institutes of Health, Public Health Service, and by grants from the Endicott-Bohn Foundation and Eli Lilly and Company. three indices - MAGE, MODD, and MBG - together help characterize the behavior of the blood glucose in that each measures something the others do not measure. Each of these indices also distinguishes among normals, stable diabetics, and unstable diabetics in our small but intensively studied samples. These same indices also serve as criteria of diabetes regulation. However, they can be calculated only from continuously obtained BG data. Therefore, we further analyzed our data to seek simpler and more convenient methods for similar characterization of patients, utilizing intermittently obtained (discrete) BG values.

\section{Résumé of Recent Work Leading to This Paper}

Clinical data concerning the subjects in this investigation and descriptions of the experimental design, biochemical methods, and data-processing procedures have been published $[1,11,12]$. The most relevant aspects of the methods and findings will be briefly summarized below.

In these studies the emphasis was on investigation of unstable diabetics who were compared with stable diabetics and normal subjects during standardized near-normal living conditions. Two programs of individually optimized therapy with insulin were used, while diet and exercise regimens remained unchanged. One program was once- or twice-daily injection of intermediate-acting insulin, with or without shortacting insulin from the same syringe. The other program 
was four-times-daily injection of short-acting insulin. The time schedule of insulin injections, meals, exercise, and sleep was consistent and precisely adhered to throughout the studies. The diet of all subjects provided four major meals of equal caloric composition and carbohydrate, protein, and fat contents. The exercise was a standardized walk three times daily during the third postprandial hour.

Each investigation culminated in a 6-day metabolic balance study after the insulin, diet, and exercise regimens had been individually standardized and optimized. For $48 \mathrm{~h}$ during this 6-day period, continuous BG monitoring was performed.

Fig. 1 shows the contrasting BG patterns of an unstable diabetic, a stable diabetic, and a normal subject. The gross qualitative differences between the diabetics and the normal as well as between the unstable and the stable diabetic are apparent. The diabetics lack the base-line stability and the rhythmicity de-

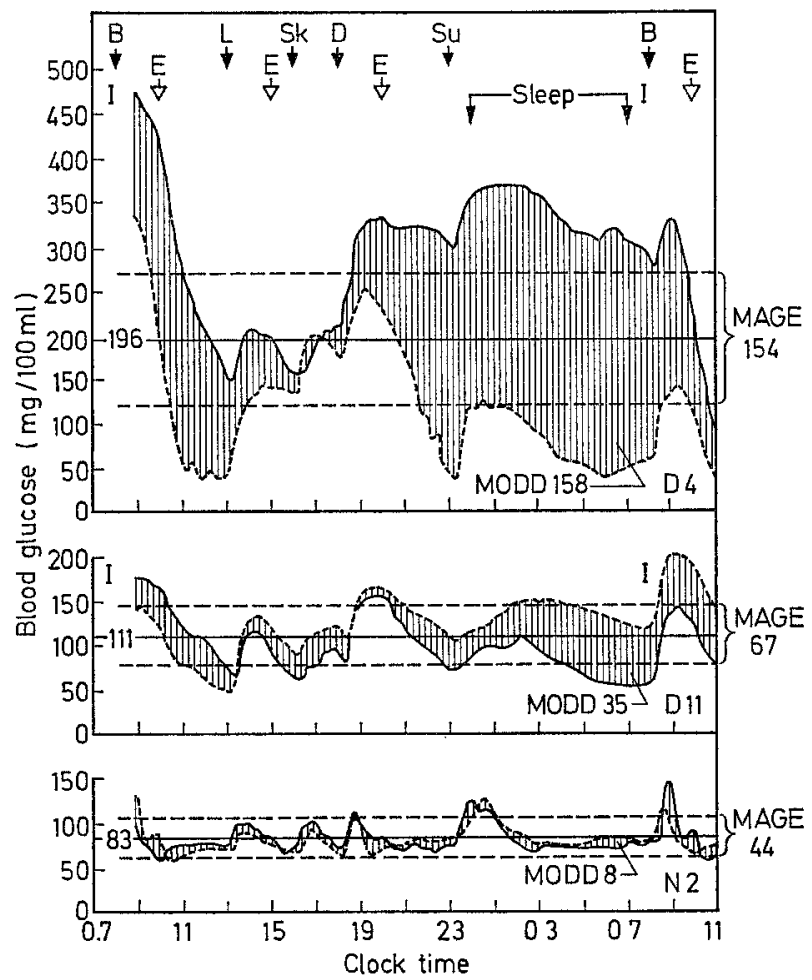

Fig. 1. Continuously sampled and analyzed blood glucose patterns over two successive $24 \mathrm{~h}$ periods in a normal subject (Bottom), a stable diabetic (Middle), and an unstable diabetic (Top). The first $24 \mathrm{~h}$ is plotted as a solid line and the second $24 \mathrm{~h}$, as a broken line. The $48 \mathrm{~h}$ mean blood glucose (MBG) is shown by the horizontal line transecting each panel; its numerical value is at the left. Mean of daily differences of paired blood glucose values (MODD) is shown by the hatched area (absolute difference between the first and second $24 \mathrm{~h}$ ); its numerical value is below. Mean amplitude of glycemic excursions (MAGE) is shown by interrupted lines bracketing the MBG; its numerical value is at the right. Symbols: $B=$ breakfast; $\mathrm{L}=$ lunch $; \mathrm{SK}=$ snack $; \mathrm{D}=$ dinner $; \mathrm{Su}=$ supper $; \mathrm{E}=$ $1 \mathrm{~h}$ of exercise; $\mathbf{I}=$ insulin monstrated by the normal subject. The unstable diabetic has the highest, the normal has the lowest, and the stable diabetic has intermediate values for the MAGE (the measure of the extent of BG excursions), the MODD (the measure of day-to-day $B G$ variation), and the $\mathrm{MBG}$ (the measure of mean $\mathrm{BG}$ ).

Fig. 1 also illustrates the experimental conditions. The daily events in the morning (between breakfast and lunch) and in the evening (between dinner and supper) were similar and, accordingly, the majority of analyses presented below are based on BG results during these times.

Conventionally utilized BG values - fasting and 1,2 , and $3 \mathrm{~h}$ after meals (breakfast and dinner) - are shown in Fig. 2 as means for groups of unstable diabetics, stable diabetics, and normals. The values were selected from the continuous BG records. There are differences between after-breakfast and after-dinner BG levels and patterns, especially among diabetics, as well as differences in BG levels and patterns between diabetics and normals.

Fig. 2 also indicates that the mean BG values at the conventionally sampled postprandial times indicate fairly well the magnitude and timing of the mean maximal BG level. Minimal BG levels, however, occur at quite different times.

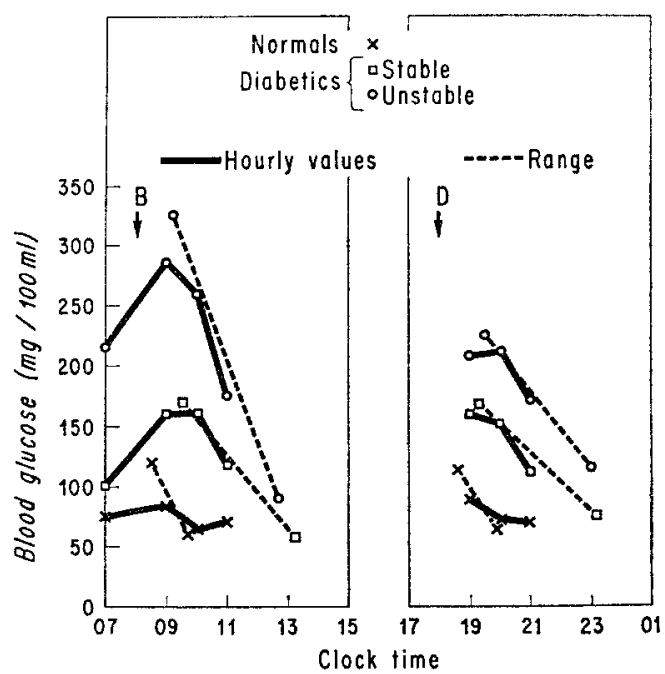

Fig. 2. Mean blood glucose values for three normal subjects, four stable diabetics, and six unstable diabetics at various times, taken from the continuously sampled and analyzed blood records. The 7 a.m. value is the fasting blood glucose concentration; the other values are at 1,2 and $3 \mathrm{~h}$ after breakfast and after dinner. The maximal and minimal blood glucose concentrations after these two meals are also indicated

\section{Methods}

Out of the large number of BG measurements available from the continuous monitoring data, we first chose to study those which were customary or had been used in other studies (fasting; 1, 2, and $3 \mathrm{~h}$ after 
breakfast; 1,2 , and $3 \mathrm{~h}$ after dinner). Also, we obtained from the continuous data the average times of occurrence of various daily maxima and minima and used BG measured at those times. This led us to add BG measured at 80 and 280 min after breakfast and after dinner (as the respective appropriate times of daily maxima and minima in diabetics). ${ }^{1}$

From this set of measurements we looked for the most promising ones to be used in place of (or as clues to the phenomena characterized by) MAGE, MBG, and MODD. In this work we chose the simple product moment coefficient of correlation as an index of association. It is easy to use and serves as a reasonable device for deciding whether one measurement was "closer" to a criterion than another. We also examined these individual measurements for their usefulness in distinguishing among the groups - normals, stable diabeties, and unstable diabetics.

\section{Results}

Portions of the above correlation studies gave the results summarized in Table 1.

MAGE was most closely associated with $\mathrm{BG}$ at 80 min after breakfast $(r=0.88)$. Also, high association was found between MAGE and the difference, BG value at 80 minus $\mathrm{BG}$ value at $280 \mathrm{~min}$ after breakfast, and the fasting $\mathrm{BG} .{ }^{2}$

MBG was highly associated with most of the $B G$ values at the selected time points. We chose $\overline{\mathrm{B}} \overline{\mathrm{G}}$, the mean of fasting $\mathrm{BG}$ and $\mathrm{BG}$ at 80 min after breakfast, for which $r=0.89$, recognizing that several others were nearly as highly correlated.

MODD was studied in conjunction with $\triangle \mathrm{BG}$, the day-to-day differences in the various $B G$ values. The best correlation appeared to be with the fasting $\mathrm{BG}$ day-to-day differences $(r=0.89)$. We call this $\triangle \mathrm{FBG}$.

The foregoing results are based on analysis of the data from stable and unstable diabetics treated with intermediate-acting insulin. The use of data from unstable diabetics treated with short-acting insulin gave

1 In diabeties, the majority of the daily maxima occurred in the morning. In normals, the daily maxima were evenly distributed during the morning, evening, and night. In all subjects, a higher proportion of daily BG minima occurred during the morning than during any other segment of the day or night.

The timing of postcibal BG maxima and minima after the start of the preceding meal was different between diabetics and normals. The mean elapsed time to BG maxima after the major daytime meals was $80 \mathrm{~min}$ (range, 77 to $91 \mathrm{~min}$ ) in diabetics and $40 \mathrm{~min}$ (range, 34 to $44 \mathrm{~min}$ ) in normals. The mean elapsed time to $B G$ minima was $280 \mathrm{~min}$ (range, 188 to $319 \mathrm{~min}$ ) in diabetics and $120 \mathrm{~min}$ (range, 104 to $144 \mathrm{~min}$ ) in normals.

2 With both programs of treatment in unstable diabetics, MAGE was closely associated with BG at 80 min after breakfast and with the difference of the $\mathrm{BG}$ values at 80 and $280 \mathrm{~min}$ after breakfast. However, with shortacting insulin treatment in unstable diabetics, MAGE was less closely associated with the fasting BG $(r=0.40)$. similar results. MAGE was most closely associated with the $\mathrm{BG}$ at $80 \mathrm{~min}$ after breakfast $(r=0.75)$. For $\mathrm{MBG}$ with FBG, $r=0.83$; for MODD with $\Delta \mathrm{FBG}, r=$ 0.83 .

Thus, by measuring the fasting $\mathrm{BG}$ value and the value at $80 \mathrm{~min}$ after breakfast, we found highly positively correlated measurements with the continuous-monitoring indices.

Table 1. Correlation coefficients of continuous-monitoring indices with selected discrete $B G$ values

\begin{tabular}{lllr}
\hline \multirow{2}{*}{ Selected BG value at } & \multicolumn{3}{c}{ Correlation coefficienta with } \\
\cline { 2 - 4 } & MAGE $^{\mathrm{a}}$ & MBGc & MODD $^{\mathrm{d}}$ \\
\hline After breakfast (min): & & & \\
60 & 0.66 & 0.82 & 0.83 \\
80 & 0.88 & 0.83 & 0.71 \\
120 & 0.48 & 0.55 & 0.03 \\
180 & 0.26 & 0.33 & -0.07 \\
280 & 0.50 & 0.71 & 0.71 \\
$80-280 \mathrm{e}$ & 0.83 & 0.64 & -0.10 \\
After dinner (min): & & & \\
60 & 0.56 & 0.76 & 0.77 \\
80 & 0.51 & 0.81 & 0.84 \\
120 & 0.50 & 0.82 & 0.77 \\
180 & 0.47 & 0.77 & 0.68 \\
280 & 0.44 & 0.87 & 0.91 \\
Fasting & 0.80 & $0.85^{\mathrm{f}}$ & 0.89 \\
\hline
\end{tabular}

a Correlation coefficients computed from data on stable and unstable diabetics treated with intermediateacting insulin $[1-3]$.

b MAGE = mean amplitude of glycemic excursions, a within-day $\mathrm{BG}$ variability index [2].

c $\mathrm{MBG}=$ mean (diurnal) $\mathrm{BG}$, an index of overall gly comia $[2]$.

d MODD $=$ mean of daily differences (of paired $B G$ values on successive days), an index of between-day $B G$ variability [3].

e Value at 80 min minus value at $280 \mathrm{~min}$

i For mean of fasting $\mathrm{BG}$ and value $80 \mathrm{~min}$ after breakfast, 0.89 .

To evaluate the potential usefulness of selecting the BG values at the chosen times, we used graphic examination and regression analysis of the relationships of the selected individual $\mathrm{BG}$ measurements with the indices from continuous BG measurements. Fig. 3 indicates an impressive association between the BG at 80 min afier breakfast and MAGE. The spreading out of the three groups of patients is no less impressive for the individual BG measurement than for MAGE. There is no overlap. The use of the regression line shown in Fig. 3 as an estimate of MAGE is good. The standard error about the regression line is $24.6 \mathrm{mg} / 100 \mathrm{ml}$ and the relative error (relative to the mean MAGE) is $21.3 \%$

Similarly $\overline{\mathrm{BG}}$ provides an adequate estimate of MBG (Fig. 4). The standard error is $23.5 \mathrm{mg} / 100 \mathrm{ml}$ and the relative error is $16.5 \%$. The spread of the three groups of patients indicates no overlap.

For MODD the situation is less satisfactory (Fig. 5). The $\triangle F B G$ does not distinguish normals from diabetics nor stable from unstable diabetics, although MODD 
itself separates the three groups without overlap. The high correlation coefficient, $r=0.89$, is partly explained by a single aberrant observation. Deleting that point reduces the coefficient to $r=0.62$. It evidently is not safe or useful to estimate MODD from the day-today difference of fasting BG. Moreover, we have not found help in estimating MODD by attempting to use day-to-day differences in other discrete BG measurements.

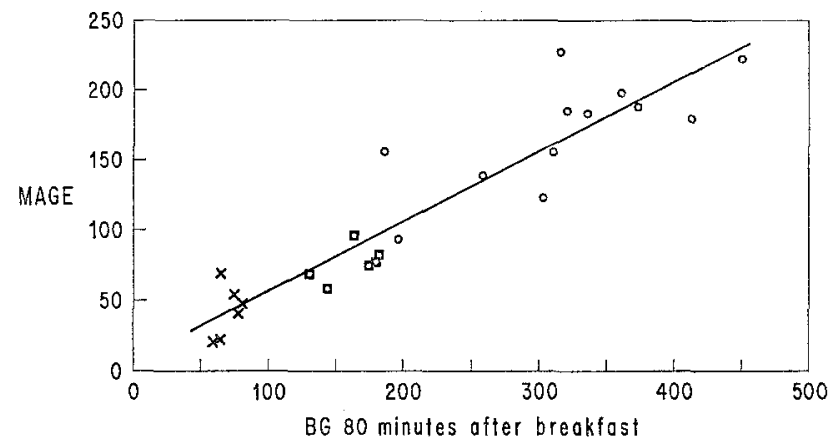

Fig. 3. Plot of $B G$ values $80 \mathrm{~min}$ after breakfast against mean amplitude of glycemic excursions (MAGE). Note good separation among normals $(x)$, stable diabetics $(\square)$, and unstable diabetics (O). For details, see text

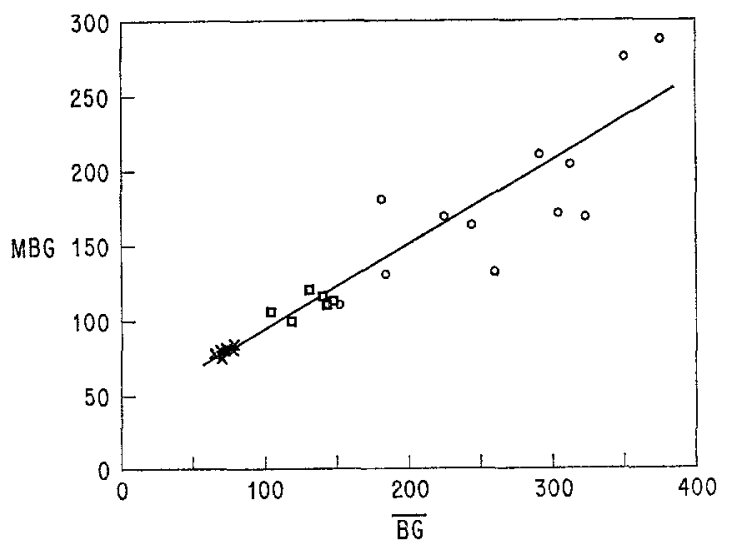

Fig. 4. Plot of $\overline{B G}$ (average of fasting $B G$ value and $B G$ value at 80 min after breakfast) against diurnal mean $B G$ (MBG). Separation of groups of subjects and symbols as in Fig. 3. For details, see text

\section{Discussion}

During treatment of diabetics, BG measurements may serve as an index of the efficacy of a particular therapeutic regimen. The most efficacious therapeutic regimen would result in minimal within-day and between-day BG variability at a near-normal mean diurnal glycemia. It would also minimize both hyperglycemic and hypoglycemic extremes.

Our results on estimating major indices of the efficacy of a therapeutic regimen fit somewhere between the routine measurements of diabetes regulation and experimental continuous $\mathrm{BG}$ monitoring. We believe that our results provide a beginning with which scientifically oriented clinicians can approach the estimation of $\mathrm{BG}$ variability in their patients. Hitherto, only during formal investigational conditions have such estimates been obtained [2, 3, 13-19]. Now this might be accomplished under conditions feasible in the setting of at least some outpatient clinics and many hospitals and at what would be to most patients an acceptable cost in the number of daily blood samplings. Of course, this approach needs critical evaluation in larger groups of patients studied over longer periods. Such further studies will, however, of necessity, be less intensive than our studies.

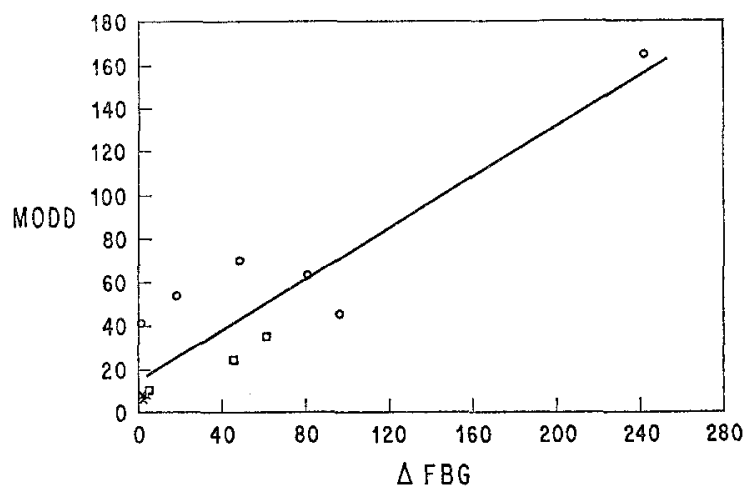

Fig. 5. Plot of $\triangle F B G$ (day-to-day differences in fasting $B G$ ) against mean of daily differences of paired $B G$ values (MOOD). Symbols as in Fig. 3. For details, see text

It is likely that, with therapeutic regimens similar to our experimental conditions, our findings may be applicable to a larger sample of all diabetics. This is suggested by our preliminary clinical experience to date with the same and additional subjects. It is possible that, even on different albeit standardized regimens, some application of our findings may be feasible. The daily maximal BG and the daily BG range are logical correlates of MAGE. Others have also found that, in diabetics on a variety of therapeutic regimens, the maximal $\mathrm{BG}$ level occurs most frequently after breakfast $[13,18,20,21]$.

The essential conditions for obtaining interpretable data are that as many as possible of the variables affecting diabetic patients (such as diet, insulin, and exercise) be standardized and that the measurements be made repeatedly on successive days with identical timing. Should unavoidable alterations of timing of therapeutic variables occur, it is probably best to alter the timing of $B G$ measurements correspondingly. For instance, if a meal is delayed by $10 \mathrm{~min}$, it is best to delay the postprandial $\mathrm{BG}$ measurements by the same interval. ${ }^{3}$ The actual time interval between the meal and the BG measurement (for example, 60 or $80 \mathrm{~min}$

$3 \mathrm{We}$ have timed postprandial $\mathrm{BG}$ measurements from the start of meals for consistency and because the timing from the start of meals is easier to standardize. 
after breakfast) is probably less important than the day-to-day consistency of diet, insulin, and exercise.

The practice of obtaining $B G$ measurements when the lowest concentrations are expected - namely, before meals and when elinical indications suggest excessive insulin action - should also be borne in mind. BG values before lunch, before dinner, and at other times when peak insulin action is suspected may provide useful information concerning possible hypoglycemia. Furthermore, with suitable quantitative urinary glucose measurements, additional useful although less direct information about $\mathrm{BG}$ variability and mean glycemia [4] may be obtained.

It is encouraging to note that a few well-timed $\mathrm{BG}$ values appear to provide useful clues (although with much loss of precision and detail) to the quantitative character of $\mathrm{BG}$ behavior. The loss of precision and detail was to be expected [19]. The disappointing lack of satisfactory clues to between-day $\mathbf{B G}$ variability obtainable from day-to-day changes in discrete $B G$ measurements further emphasizes the wealth of information provided by continuous $\mathrm{BG}$ monitoring $[1-3$, $19,20]$. Yet it is with reference to between-day variability that continuous BG monitoring has its principal drawback: it is limited, for various reasons, to 48 to $72 \mathrm{~h}[1,12,19]$. Because of the importance of betweenday $\mathrm{BG}$ variability $[13,14,18]$, better means of assessing this aspect of $\mathrm{BG}$ variability need to be found.

There is clearly a need for systematic assessment of the adequacy of diabetes regulation. The lack of satisfactory BG criteria applicable to the assessment of diabetes regulation under long-term, real-life conditions has hampered objective appraisal of the results of therapy. This in turn has made correlation between adequacy of diabetic regulation and other phenomena, including the incidence and progression of diabetic complications, virtually impossible [22].

\section{References}

1. Molnar, G.D., Ackerman, E., Rosevear, J.W., Gatewood, L.C., Moxness, K. E.: Continuous blood glucose analysis in ambulatory fed subjects. I. General methodology. Mayo Clin. Proc. 43, 833-851 (1968)

2. Service, F.J., Molnar, G.D., Rosevear, J.W., Ackerman, E., Gatewood, L.C., Taylor, W.F.: Mean amplitude of glycemic excursions, a measure of diabetic instability. Diabetes 19, 644-655 (1970)

3. Molnar, G.D., Taylor, W.F., Ho, M.M.: Day-to-day variation of continuously monitored glycemia: a further measure of diabetic instability. Diabetologia $8,342-348(1972)$

4. Service, F.J., Molnar, G.D., Taylor, W.F.: Urine glucose analyses during continuous blood glucose monitoring. J. Amer. med. Ass. 222, 294-298 (1972)

5. Krall, L.P., Joslin, A.P.: General plan of treatment and diet regulation. In: Joslin's Diabetes Mellitus, $p$. 258, 11 th edition. (Ed. Marble, A., White, P., Bradley, R.F., Krall,L.P.). Philadelphia: Lea and Febiger 1971

6. Marble, A.: Insulin in the treatment of diabetes. In: Joslin's Diabetes Mellitus, p. 290, 11 th edition. (Ed.
Marble, A., White, P., Bradley, R.F., Krall, L.P.). Philadelphia: Lea and Febiger 1971

7. White, P., Graham, C.A.: The child with diabetes. In : Joslin's Diabetes Mellitus, p. 341-345, 11 th edition. (Ed. Marble, A., White, P., Bradley, R.F., Krall, L. P.). Philadelphia: Lea and Febiger 1971

8. Colwell, A.R., Sr.: Clinical use of insulin. In : Diabetes Mellitus: Theory and Practice, p. 624-637. (Ed. Ellenberg, Rifkin, H.). New York: McGraw-Hill Book Company 1970

9. Knowles, H.C., Jr.: Control of diabetes and the progression of vascular disease. In: Diabetes Mellitus: Theory and Practice, p. 666-673. (Ed. Ellenberg, M., Rifkin, H.). New York: McGraw-Hill Book Company 1970

10. Sussman, K.E.: Juvenile-type diabetes and its complications: theoretical and practical considerations, p. 68-70. Springfield, Illinois: Charles C. Thomas, Publisher 1971

11. Service, F.J., Molnar, G.D., Rosevear, J.W., Ackerman, E., Taylor, W.F., Cremer, G.M., Moxness, K.E.: Continuous blood glucose analysis in ambulatory fed subjects. IT. Eiffects of anticoagulation with heparin. Mayo Clin. Proc. 44, 466-477 (1969)

12. Rosevear, J.W., Pfaff, K.J., Service, F.J., Molnar, G.D., Ackerman, E.: Glucose oxidase method for continuous automated blood glucose determination. Clin. Chem. 15, 680-698 (1969)

13. Czyzyk, A., Ponikowska, I.: La signification des valeurs particulières de la glicémie dans le cycle glycemique journalier. Acta diabet. lat. 4, 374-385 (1967)

14. Izzo, J.L., Crump, S.L.: A clinical comparison of modified insulins. J. clin. Invest. 29, 1514-1527 (1950)

15. Schöne, G., Zimmer, H.: Über Blutzuckertageskurven bei Gesunden und Zuckerkranken. Klin. Wschr. 14, 1672-1677 (1935)

16. Hallas-Møller, K.: The lente insulins. Diabetes 5, 7$12(1956)$

17. Bressler, R., Galloway, J.A.: Insulin treatment of diabetes mellitus. Med. Clin. N. Amer. 55, 861-876 (1971)

18. Schlichtkrull, J., Munck, O., Jersild, M. : The M-value, an index of blood-sugar control in diabetics. Acta med. scand. 177, 95-102 (1965)

19. Mirouze, J., Satingher, A., Sany, C., Jaffiol, C.: Coefficient d'efficacité insulinique: coefficient $M$ de Schlichtkrull corrigé et simplifié par la technique de l'enregistrement glycémique continu. Diabete 11, $267-273(1963)$

20. Jersild, M.: La glycémie post-prandiale des diabétiques. Diabete 14, 225-227 (1966)

21. Sindoni, A., Jr.: Fasting blood sugar vs. postprandial blood sugar as observed in normal individuals, medical (non-diabetic) patients, and patients with diabetes; special references to: plain, protamine zinc and globin insulins, compatible hyperglycemia and arteriosclerosis. Amer. J. dig. Dis. 13, 178-192 (1946)

22. Kaplan, M.H., Feinstein, A.R.: A critique of methods in reported studies of long-term vascular complications in patients with diabetes mellitus. Diabetes 22 , $160-174(1973)$

Dr. G.D. Molnar

Dept. of Endocrinology

and Internal Medicine

Mayo Clinic

Rochester

Minnesota 55901

USA 